Research Journal of Applied Sciences 6 (3): 209-212, 2011

ISSN: $1815-932 \mathrm{X}$

(C) Medwell Journals, 2011

\title{
Measurement and Studied on Some Plasma Parameters in the Edge of IR-T1 Tokamak
}

\author{
${ }^{1}$ A. Razmara, ${ }^{1} \mathrm{P}$. Khorshid and ${ }^{2} \mathrm{M}$. Ghoranneviss \\ ${ }^{1}$ Department of Physics, Islamic Azad University, Mashhad Branch, Mashhad, Iran \\ ${ }^{2}$ Plasma Physics Research Center, Science and Research Branch, \\ Islamic Azad University, Tehran, Iran
}

\begin{abstract}
The Plasma resistivity has been measured in the edge plasma of IR-T1 tokamak. The fundamental plasma parameters of electron Temperature $\left(\mathrm{T}_{e}\right)$ and electron density $\left(\mathrm{n}_{\mathrm{e}}\right)$ are measured simultaneously for calculating the radial profile of vertical and parallel resistivity using IV characteristics of a moveable single Langmuir probe. Since the effective charge $\left(Z_{\text {efff }}\right)$ and $T_{e}$ together define the plasma resistivity, $Z_{\text {eff }}$ considered for resistivity measurement in Spitzer model. The results have shown that electron density increased from edge to core of plasma column, as the parallel electrical resistivity of the plasma decreases with increasing temperature. As temperature of plasma raised, resistivity drops rapidly so reduction of resistivity with increasing temperature could leads to prolonging of the duration of the plasma discharge.
\end{abstract}

Key words: Tokamaks, resistive $\mathrm{MHD}$, electron collisions, impurities in plasmas, ohmic heating, electric discharges

\section{INTRODUCTION}

Any realistic plasma will have a density gradient and the plasma will tend to diffuse toward regions of low density. We assume that the plasma is weakly ionized so that charge particles collide primarily with neutral atoms rather than with one another. As the plasma spreads out as a result of pressure gradient and electric field forces, the individual particles undergo a random walk, colliding frequently with the neutral atoms. When plasma consists of just electrons and ions, all collisions are coulomb collisions among charge particle (Trintchouk et al., 2003) (Chen, 1984). If a uniform steady electric field is imposed on plasma this electric field will accelerate the ions and electrons in opposite directions. The accelerated particles will collide with other particles and this fractional drag will oppose the acceleration. Resistivity is determined by the collisional drag on electrons moving against the background of ions. Suppose that an electric field E exists in a plasma and that the current that it drives is all carried by the electrons which are much more mobile than the ions. Then in steady state the electron equation of motion changes so that $E=\eta \mathrm{J}$ is ohm's low and $\eta$ is the resistivity. The transverse or cross field resistivity was calculated by Spitzer as the rate of momentum transfer from electrons to ions through collisions in a resistive magnetohydrodynamics (Trintchouk et al., 2003) (De Blank, 2006):

$$
\eta_{\perp}=1.03 \times 10^{-4} \mathrm{~T}_{e}^{-3 / 2} \mathrm{Z} \ln \Lambda \text { (ohm.m) }
$$

where, the electron temperature $T_{e}$ is in electron volts, $Z$ is the ion atomic mass and $\ln \Lambda$ is the coulomb logarithm. Also parallel resistivity defined by Darian et al. (2006) and Goldston and Rutherford (1995):

$$
\eta_{\text {Spitzer }}=\eta_{\|}=5.24 \times 10^{-5} \frac{Z \ln \Lambda}{T_{e}^{3 / 2}}(\text { ohm.m) }
$$

$\ln \Lambda$ is coulomb logarithm which is a term that accounts for the multiple small angle collisions in the plasma and Debye shielding.

$$
\Lambda=1.5492 \times 10^{13} \frac{\mathrm{T}_{\mathrm{e}}^{\frac{3}{2}}}{\mathrm{Z}^{2} \sqrt{\mathrm{n}_{\mathrm{e}}}}
$$

$\ln \Lambda$ is 15.75 for IR-T1 tokamak. On the other hand, impurities in plasma are one of important factors of instabilities. For example impurities prevent plasma from heating. We have defined $Z_{\text {eff }}$ as the ratio of the measured plasma resistivity $\eta_{p}$ to the theoretical resistivity $\eta_{\| \mid}$. For ohmic input power, plasma resistivity is:

$$
\eta_{p}=\frac{r^{2} V_{1}}{2 R I_{p}}
$$

Corresponding Author: A. Razmara, Department of Physics, Islamic Azad University, Mashhad Branch, Mashhad, Iran 




Fig. 1: Time evolution of plasma current and effective charge

Where:

$\mathrm{V}_{1}=$ Loop voltage

$\mathrm{I}_{\mathrm{p}}=$ The plasma current

$\mathrm{R}=$ The major radius of tokamak chamber

By definition of $Z_{\text {eff }}$ the value of $\eta_{p}$ could be calculated. The time evolution of $Z_{\text {eff }}$ from a typical electric discharge of IR-T1 tokamak has been shown in Fig. 1.

Experimental set-up and diagnostics: $\mathbb{R}-\mathrm{T} 1$ tokomak is an ohmically heated air core tokamak with a major radius of $\mathrm{R}=0.45 \mathrm{~m}$ and a minor radius of $\mathrm{a}=0.125 \mathrm{~m}$ defined by two poloidal stainless-steel limiters. The vacuum chamber has a circular cross-section with two toroidal breaks and a minor radius of $b=0.15 \mathrm{~m}$. Toroidal magnetic field is equal to $B_{t} \sim 0.6-0.8 \mathrm{~T}$, plasma current is $\mathrm{I}_{\mathrm{p}} \sim 25-30 \mathrm{kA}$, averaged electron density in hydrogen is $0.5-1.5 \times 10^{19} \mathrm{~m}^{-3}$, plasma discharge duration is $t_{d} \sim 30 \mathrm{msec}$ and electron temperature is $T_{e}(0) \sim 150-180 \mathrm{eV}$. A single Langmuir probe was used to measure spatial and temporal evolutions of electron temperature and density. The probe is connected to the power supply where its potential is varied continuously over a range from negative to positive potential with respect to the plasma potential to obtain the ion and electron current. The current, I is determined as a function of the applied probe voltage $\mathrm{V}_{\text {app }}$. This relation $\mathrm{I}=\mathrm{f}\left(\mathrm{V}_{\text {app }}\right)$ is called the probe characteristic or the I-V characteristic. The general appearance of the I-V characteristic for a Langmuir probe is shown in Fig. 2.

Langmuir probes use in low temperature plasmas to measure the plasma density, electron temperature and plasma potential. A Langmuir probe inserted into a plasma and electrically biased with respect to a refrence electrode to collect electron and ion currents. Suppose that a probe is present in a plasma of comparable electron and ion temperatures. The total electric current from a probe of area A dominated by electrons because the ion speed will then be smaller than the electron speed (electron mass is larger than ion mass):

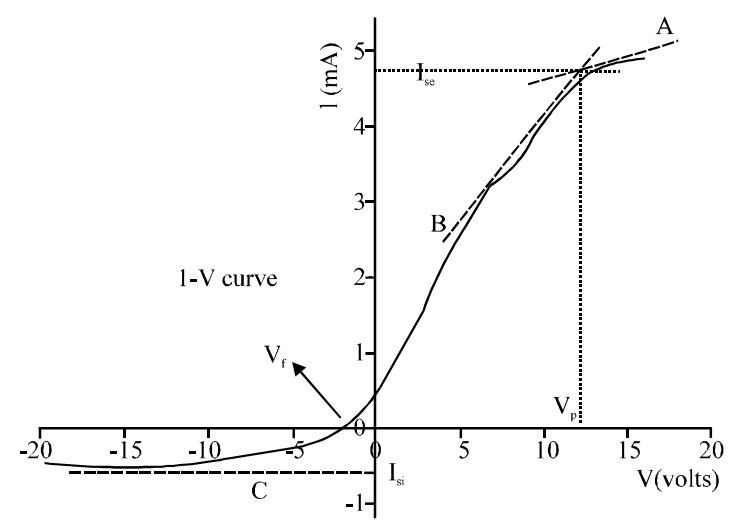

Fig. 2: The I-V characteristic Langmuir probe during a typical discharge of IR-T1 tokamak

$$
\mathrm{I}=-\mathrm{eA}\left(\frac{1}{4} \mathrm{n}_{\mathrm{i}} \overline{\mathrm{V}_{\mathrm{i}}}-\frac{1}{4} \mathrm{n}_{\mathrm{e}} \overline{\mathrm{V}_{\mathrm{e}}}\right) \approx \frac{1}{4} \mathrm{e} A n_{\mathrm{e}} \overline{\mathrm{V}}_{\mathrm{e}}>0
$$

The probe would thus emit a net positive current. If probe potential is equal to space potential, space potential is equal with potential at $I=I_{s e}$. If $V_{B}>V_{P}$ the electron current connot increase any further, this is because the electron current is maximized. This region (A) is known as electron saturation and I here is equal to the electronsaturation current. With decreasing the probe potential, $\mathrm{V}<\mathrm{V}_{\mathrm{P}}$, the probe is negative with respect to the surrounding plasma and this causes to reflect part of the impacting electrons (region B). Eventually in this potential, I reduce to small fraction of saturation current. The total current is zero when $\mathrm{I}_{\mathrm{i}} \approx \mathrm{I}_{\mathrm{e}}$. This phenomena happen when the probe potential is equal to floating potential $\mathrm{V}_{\mathrm{f}}$. Decreasing the potential further (entering region $\mathrm{C}$ ) probe with constant rate, enable to collected ions. This is ion saturation current, $I=I_{\mathrm{s}}($ Merlino, 2007). Radial profile of electron density and electron temperature has been calculated by Langmuir probe, behind and in front of the fixed poloidal limiter from $\mathrm{r}=10.5-13.5 \mathrm{~cm}$. The equation:

$$
I_{\text {probe }}=I_{s a t}^{+}\left[1-\exp \left(\frac{V_{p r}-V_{f}}{k T_{e}}\right)\right]
$$

is fitted to the $I-V$ date which $T_{e}$ is obtained and also $n_{e}$ achived by Merlino (2007) and Popescu et al. (2007):

$$
\mathrm{I}_{\mathrm{is}}=\mathrm{I}_{\mathrm{Bohm}}=0.6 \mathrm{en}_{\mathrm{i}} \sqrt{\frac{\mathrm{kT}_{\mathrm{e}}}{\mathrm{m}_{\mathrm{i}}} \mathrm{A}}
$$

The results have been shown in Fig. 3. The radial profile of electron temperature and electron density have been shown in Fig. 3. It can deduced that the electron 

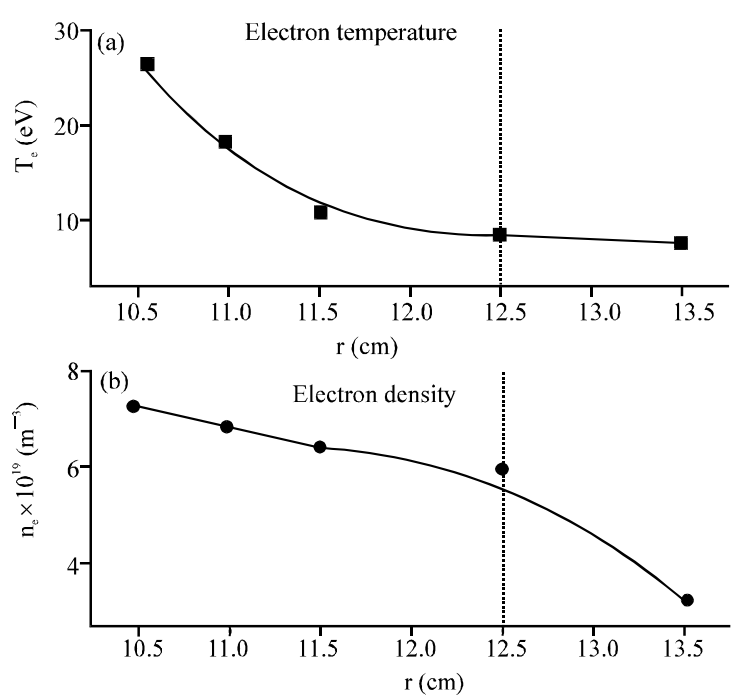

Fig. 3: a) The radial profile of electron temperature and b) the radial profile of electron density

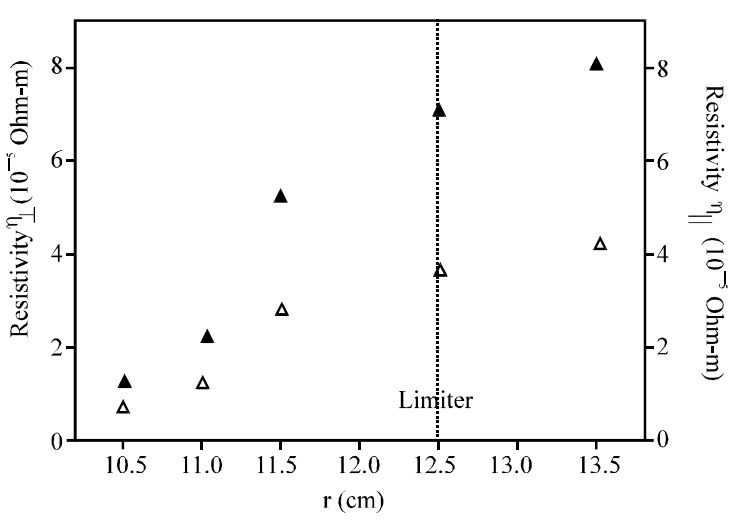

Fig. 4: The radial profile of parallel and vertical plasma resistivity

temperature and electron density are increasing from edge to core of plasma so according to Eq. 2 the plasma resistivity will decrease by increasing electron temperature. In other hand, when electron temperature increases, the collision frequency is decrease. Collisions between electrons and ions in plasma will prevent the accelerations of electrons in response to an electric field. Without such collisions, electrons would be accelerated indefinitely by an applied electric field so that an infinitesimal voltage would be sufficient to drive a large current through plasma. Collisions between electrons and ions cause to limit the current that can be driven by an electric field. Also resistivity may be expressed in terms of the electron-ion collision frequency (Goldston and Rutherford, 1995):

$$
v_{\mathrm{ei}}=\left(\frac{\mathrm{ne}^{2}}{\mathrm{~m}}\right) \eta
$$

So we can deduce from above sentences that when electron temperature increases, collision frequency decrease therefore plasma resistivity decreases. The Fig. 4 shows the calculated radial profile of parallel and vertical plasma resistivity it decreased from edge to core. We found that transverse resistivity for electrons are higher than the parallel resistivity by a factor of 1.96 , the results have been compared with other tokamak plasmas and it is similar to TCABR and CASTOR tokamak. The result have shown that when electron temperature increased, parallel electrical resistivity decreases, this could leads to prolonging of the plasma discharge.

\section{CONCLUSION}

In this study researchers obtained the plasma resistivity profile using parameters of plasma such as $n_{e}$ and $\mathrm{T}_{e}$ calculated by I-V characteristics of moveable single Langmuir probe in the edge of IR-T1 tokamak. The results showed that electron temperature decrease from core to edge as for electron density is. Plasma resistivity is proportional to $\mathrm{T}_{\mathrm{e}}^{-3 / 2}$ so resistivity increase from center to edge of plasma. As temperature of plasma raised, resistivity drops rapidly so plasma at very high temperatures is collision-less i.e., their resistivity is negligible. Reduction of resistivity with increasing temperature could leads to prolonging of the duration of the plasma discharge. Effective charge may be important to measurement of resistivity in high plasma impurity so that it can prevent the plasma for ohmicaly heating.

\section{ACKNOWLEDGEMENTS}

The researchers would like gratefully to acknowledge IR-T1 team for their technical assistance and researcher A.R. thanks Dr. Z. Emami for her helpful discussions.

\section{REFERENCES}

Chen, F.F., 1984. Introduction to Plasma Physics and Controlled Fusion. Plenum Press, Los Angeles.

Darian, M.M., A. Hojabri, M. K. Salem and M. Ghoranneviss, 2006. The influence of resonance helical field on the zeff and impurity radiation in IR-T1 Tokamak. J. Fusion Energy, 25: 241-244. 
De Blank, H.J., 2006. MHD instabilities in tokamaks. Trans. Fusion Sci. Technol., 49: 118-130.

Goldston, R.J. and P.H. Rutherford, 1995. Introduction to Plasma Physics. IOP Physics Publishing Ltd., Philadelphia, pp: 491.

Merlino, R.L., 2007. Understanding Langmuir probe current-voltage characteristics. Am. J. Phys., 75: 1078-1085.
Popescu, S., Y. Ohtsu and H. Fujita, 2007. Langmuir probe data analysis for a magnetized inductive radiofrequency discharge. J. Applied Phys., 102: 093302093307.

Trintchouk, F., M. Yamada, H. Ji, R.M. Kulsrud and T.A. Carter, 2003. Measurement of the transverse Spitzer resistivity during collisional magnetic reconnection. Phys. Plasmas, 10: 319-322. 